\title{
An Attempt on the Calculation of Relativistic Potential Energy Curves: Noble Gas Difluorides *
}

\author{
R. Euler, B. Fricke, T. Morović, and W.-D. Sepp \\ Fachbereich Physik der Gesamthochschule, Kassel, Federal Republic of Germany \\ A. Rosén \\ Physics Department, Chalmers University of Technology, Göteborg, Sweden
}

Received July 12, 1980

Total energy SCF calculations were performed for noble gas difluorides in a relativistic procedure and compared with analogous non-relativistic calculations. The discrete variational method with numerical basis functions was used. Rather smooth potential energy curves could be obtained. The theoretical $\mathrm{Kr}-\mathrm{F}$ and $\mathrm{Xe}-\mathrm{F}$ bond distances were calculated to be 3.5 a.u. and 3.6 a.u. which should be compared with the experimental values of 3.54 a.u. and 3.7 a.u. Although the dissociation energies are off by a factor of about five it was found that $\mathrm{ArF}_{2}$ may be a stable molecule. Theoretical ionization energies for the outer levels reproduce the experimental values for $\mathrm{KrF}_{2}$ and $\mathrm{XeF}_{2}$ to within $2 \mathrm{eV}$.

\section{Introduction}

$\mathrm{Ab}$ initio non-relativistic SCF calculations within various approximations have become an important tool in physics and chemistry of small molecules for low $Z$ elements. In molecules which include heavier atoms, relativistic effects are expected to become important. From the detailed comparison of non-relativistic and relativistic atomic calculations one knows very well the effects of strong relativistic contraction of all $s$ and $p_{1 / 2}$ wavefunctions and the expansion of the wavefunctions with large angular momenta due to the indirect relativistic effects. Besides many other examples it has been found that the two-valency in lead or the difference in chemistry between silver and gold are mainly of relativistic origin [1]. Similar effects, typical for relativistic influences, are also expected for molecules including heavy atoms [2].

The development of a relativistic Dirac-Slater code $[3,4]$ which is briefly described in Chap. 2 now makes it possible to calculate wavefunctions and

\footnotetext{
* Partially supported by GSI Darmstadt
}

energy eigenvalues of small molecules including heavy atoms. Up to now, all interpretations of these calculations have been performed by using the energy eigenvalues or wavefunctions in order to discuss relativistic influences on absorption spectra or level structure of outer electrons [4-8]. Ionization energies are discussed up to now only within the so-called transition state procedure [9]. Due to the numerical inaccuracy of the method involved, only one attempt [10] has been undertaken earlier to calculate directly relativistic total energies for a low $Z$ molecule. In this paper we present our first calculations of $a b$ initio relativistic potential energy curves for heavy systems with the aim to get dissociation energies and bond distances. As examples we have chosen various noble gas difluorides. The reason for this choice was the theoretical importance of these compounds which did not exist until the early 60's [11-12]. Furthermore, very elaborate non-relativistic calculations have been published [13-14]. The only noble gas difluorides which are known are $\mathrm{KrF}_{2}$ and $\mathrm{XeF}_{2}$ with a bond length of 3.5 a.u. [15,16] and 3.7 a.u. [17] and bond 
energies of $1.01 \mathrm{eV}$ [18] and $2.78 \mathrm{eV}$ [19], respectively.

In Chap. 3 we study the results of the relativistic DS calculation of $\mathrm{XeF}_{2}, \mathrm{KrF}_{2}, \mathrm{ArF}_{2}$ and $\mathrm{NeF}_{2}$. To investigate possible relativistic influences we compare with non-relativistic calculations using the same principal method. The results are analysed in order to understand the accuracy of the Dirac Slater model used $[3,4]$ as well as the large number of numerical and computational problems which have to be solved until this method can be used in general.

\section{Method}

The dissociation energy of an atom in a molecule with distance $R$ to the nearest neighbour is given by the difference $\Delta E(R)$ of the total system energy $E^{\text {tot }}(R)$ for this particular $R$ and the sum of the total energies of the separated atoms $E^{\text {tot }}(R \rightarrow \infty)$ and $E^{\text {tot }}$ for the rest of the molecule. In our case we are interested in the dissociation of the whole noble gas difluorides; and $R$ describes the distance of the two fluoride nuclei with respect to the central nucleus. Then $\Delta E(R)$ can be written as

$\Delta E(R)=E^{\mathrm{tot}}(R)-\sum_{i=1}^{3} E_{i}^{\mathrm{tot}}(\infty)$.

The total statistical energy of an electronic system with the one-electron density $\rho(\mathbf{X} ; R)$ is given by Slater [20]

$E^{\mathrm{tot}}(R)=\sum_{i} \varepsilon_{i}(R)-\frac{1}{2} \int \rho(\mathbf{X} ; R) V_{c}(\mathbf{X} ; R) d \mathbf{X}$

$-\frac{1}{4} \int \rho(\mathbf{X} ; R) V_{\mathrm{ex}}(\mathbf{X} ; R) d \mathbf{X}+V_{\text {nuc }}(R)$.

$V_{c}(\mathbf{X} ; R)$ and $V_{\mathrm{ex}}(\mathbf{X} ; R)$ denote the direct and the exchange part of the electronic potential, respectively. $V_{\mathrm{ex}}(\mathbf{X} ; R)$ is the local exchange approximation given by

$V_{\mathrm{ex}}(\mathbf{X} ; R)=-3 \alpha[3 / 8 \pi \rho(\mathbf{X} ; R)]^{1 / 3}$

as originally derived by Slater [21]. For the exchange parameter $\alpha$ we always use the value 0.7 . The last term in (2), $V_{\text {nuc }}(R)$, represents the electrostatic repulsion between the nuclei of the molecule. $\mathbf{X}$ represents the generalized coordinates of an electron at the position $\mathbf{r}$ with the spin coordinate $s$. The one-electron Dirac-Slater or Hartree-Fock-Slater equation for each particular distance $R$

$h(\mathbf{X} ; R) \psi_{i}(\mathbf{X} ; R)=\varepsilon_{i}(R) \psi_{i}(\mathbf{X} ; R)$

can be derived by applying the variational principle to the statistical total energy. The resulting eigen- values $\varepsilon_{i}$ have then to be summed up to the Fermi level in (2).

We solve the one-electron equation (4) using the discrete variational method $[22,23]$ to find the molecular wavefunctions which are approximated by linear combinations of symmetry orbitals constructed from numerical atomic SCF basis functions.

The basis set for the noble gas atoms has been generated in the $n p^{6}$ configuration for the free atomic problem. The molecular potential was determined in the successive iteration from Mulliken gross orbital populations for the basis functions. These populations were spherically averaged and the molecular potential was constructed from the spherical charges on the constituent atoms. This method is referred to as the self-consistent charge (SCC) procedure [24]. Self-consistency was obtained when the input and output orbital charges were equal. This procedure does not imply spherical symmetry of the molecular potential as for example in the "muffin-tin" form used in the multiple scattering method (MSX $\alpha$ ). The SCC procedure is on the other hand an approximation to the more accurate DV-X $\propto$ method used by the Amsterdam group $[25,26]$.

\section{Results and Discussions}

The total energy is not achieved easily in an actual numerical calculation using the numerical integration method described above because the convergence of the SCF procedure is still very slow. One always has to have in mind that the distribution of 2,000 to 5,000 points within the whole space is not very much for a linear molecule like $\mathrm{F}-\mathrm{Xe}-\mathrm{F}$. So the total energy still varies by $2-3 \mathrm{eV}$ for $\mathrm{NeF}_{2}, \mathrm{ArF}_{2}$ and by $15 \mathrm{eV}$ for $\mathrm{KrF}_{2}, \mathrm{XeF}_{2}$ and $\mathrm{RnF}_{2}$ for a change of integration points from 2,000 to 5,000 . Due to the very heavy atoms in the molecules calculated, the total energies of course are very large, which means, that in a full SCF procedure with no frozen core approximation the accuracy of the calculation also has to increase drastically in order to get results for chemical interpretations. (The total energy of $\mathrm{KrF}_{2}$ is $82 \mathrm{keV}$ and for $\mathrm{XeF}_{2}$ it is already more than $200 \mathrm{keV}$.) Baerends and $\operatorname{Ros}[25,26]$ therefore calculate only the exchange term in (2) numerically with the DVM method and evaluate the other terms analytically using the properties of the Slater type orbital (STO) basis functions. They found that it was otherwise hardly possible to calculate dissociation energies unless very many sample points were used [27]. In order to overcome the numerical problems in this work the potential energy curves are derived in the following way. In the first step the total energies are calculated 


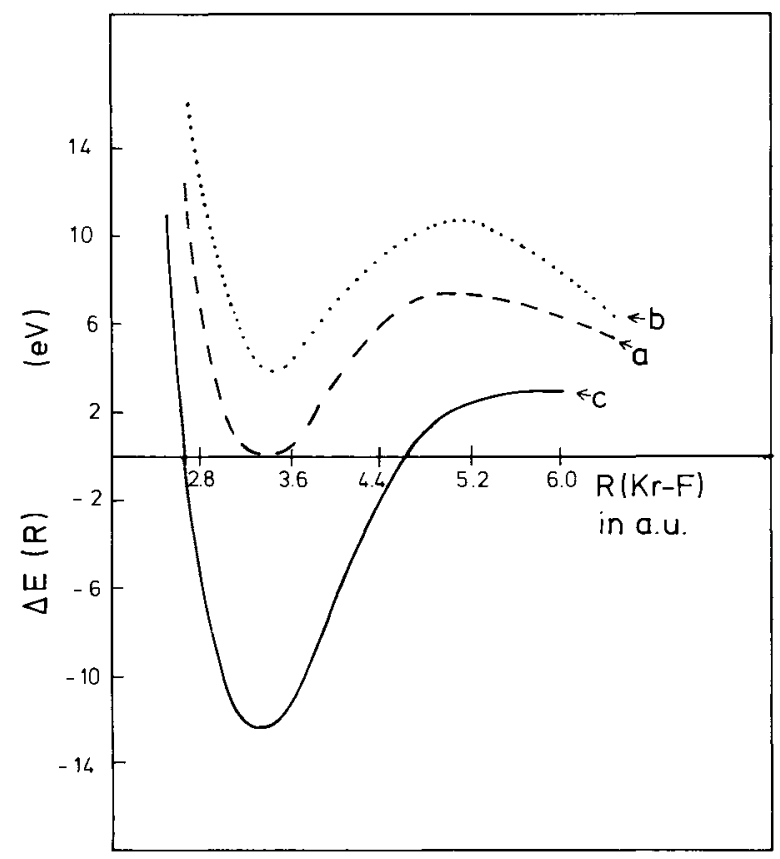

Fig. 1. Potential energy curves calculated for $\mathrm{KrF}_{2}$ with a) nonrelativistic method and minimal basis set, b) relativistic method and minimal basis set, c) non-relativistic method and extended basis

directly according to (2) for the molecule within our numerical code. From these values we subtract the total energies of the three atoms but not within an independent atomic calculation, but calculated with the same molecular code where the nuclear charges of the noble gas atom or $F$, respectively, are set equal
Table 1. Calculated and experimental dissociation energies and bond distances for $\mathrm{NeF}_{2}, \mathrm{ArF}_{2}, \mathrm{KfF}_{2}$ and $\mathrm{XeF}_{2}$

\begin{tabular}{|c|c|c|c|c|c|c|c|}
\hline & & \multirow{2}{*}{$\begin{array}{l}\mathrm{NeF}_{2} \\
\text { rel }\end{array}$} & \multirow{2}{*}{$\begin{array}{l}\mathrm{ArF}_{2} \\
\text { rel }\end{array}$} & \multicolumn{2}{|c|}{$\mathrm{KrF}_{2}$} & \multicolumn{2}{|c|}{$\mathrm{XeF}_{2}$} \\
\hline & & & & rel & nonrel & rel & nonrel \\
\hline Calcul. & $\begin{array}{l}R_{B} / \text { a.u. } \\
E_{D} / \mathrm{eV}\end{array}$ & $\begin{array}{l}3.0 \\
0.3\end{array}$ & $\begin{array}{l}3.50 \\
2.95\end{array}$ & $\begin{array}{l}3.45 \\
6.60\end{array}$ & $\begin{array}{l}3.40 \\
7.10\end{array}$ & $\begin{array}{r}3.61 \\
12.20\end{array}$ & $\begin{array}{r}3.52 \\
13.10\end{array}$ \\
\hline Exp. & $\begin{array}{l}R_{B} / \text { a.u. } \\
E_{D} / \mathrm{eV}\end{array}$ & $\begin{array}{l}- \\
-\end{array}$ & $\begin{array}{l}- \\
-\end{array}$ & & $\begin{array}{l}.54 \\
.01\end{array}$ & & $\begin{array}{l}3.74 \\
2.8\end{array}$ \\
\hline
\end{tabular}

to zero. This procedure is expected to reduce the numerical errors as far as possible. The results presented in Fig. 1 for the potential energy curves for various calculations of $\mathrm{KrF}_{2}$ show a rather smooth behaviour. Curve $a$ and $b$ give the results using a minimal basis set consisting of only occupied atomic orbitals for the free atoms. In curve $c$ the basis set is enlarged by $4 d, 5 s$ and $5 p$ wavefunctions of atomic $\mathrm{Kr}$, obtained by superimposing upon the self-consistent potential for the free atoms a potential well of radius 20 a.u. and a depth of $2.7 \mathrm{eV}$. The large change compared with the minimal basis set calculation shows that the result is largely basis set dependent, but the trend of the potential energy curve especially at large distances is in the right direction. It is wellknown from non-relativistic calculations that a larger basis increases the minimum whereas the non-physical maximum in the outer region disappears [13, 14]. The same trend can be observed here. On the other hand an increase in the number of grid points has no large effect on the potential energy curve. So
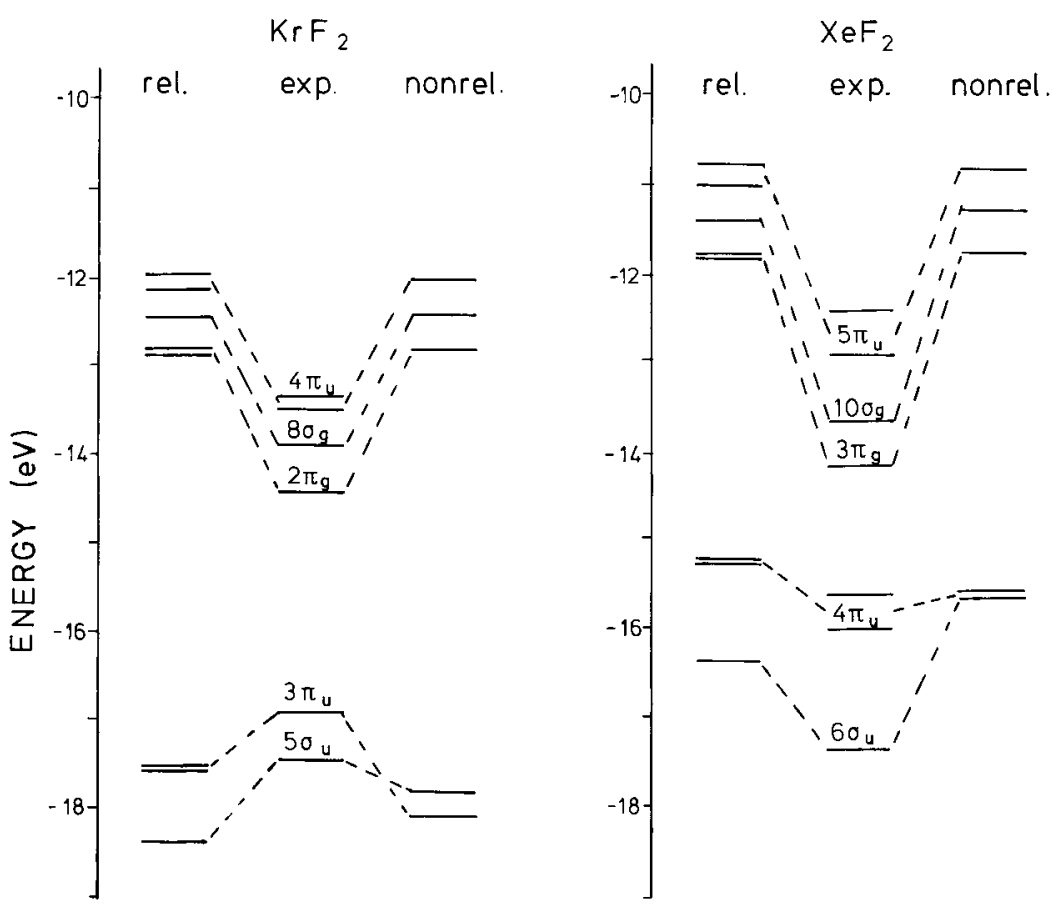

Fig. 2. A comparison of theoretical and experimental ionization energies for $\mathrm{KrF}_{2}$ and $\mathrm{XeF}_{2}$ 
we can expect that the inclusion of additional atomic wavefunctions leads to the correct behaviour of the potential energy curve for large distances.

The main discrepancy up to now is that the absolute minimum is by far too deep. On the other hand the location of the minimum itself is very near to the experimental value. Table 1 presents the results of the calculated dissociation energies and bond distances for $\mathrm{NeF}_{2}, \mathrm{ArF}_{2}, \mathrm{KrF}_{2}$ and $\mathrm{XeF}_{2}$ to compare with the experimental results of $\mathrm{KrF}_{2}$ and $\mathrm{XeF}_{2}$. Although - as stated above - the calculated dissociation energies differ very much from the experimental ones, a clear trend can be seen. This allows us to predict by extrapolation $E_{D} \approx 0.5 \mathrm{eV}$ as the value for the dissociation energy of $\mathrm{ArF}_{2}$. The theoretical potential energy curve for $\mathrm{NeF}_{2}$ does not show a clear minimum so that it cannot be expected that $\mathrm{NeF}_{2}$ may exist. A comparison between experimental $[28,29]$ and theoretical ionization energies for the outer levels for $\mathrm{KrF}_{2}$ and $\mathrm{XeF}_{2}$ is presented in Fig. 2. The theoretical values reproduce the experimental ones within $2 \mathrm{eV}$. The relative position within a group of levels of course is much better. Figure 2 also shows that only relativistic calculations reproduce the correct number and order of the levels, whereas the non-relativistic calculations are considerably worse. The theoretical value of the spin-orbit splitting for the $5 \pi_{u}$ level in $\mathrm{XeF}_{2}$ is $0.2 \mathrm{eV}$ compared with the experimental value of $0.5 \mathrm{eV}$. The $4 \pi_{u}$ level is mainly of $\mathrm{F}-2 p$ character, and the theoretical value of $0.1 \mathrm{eV}$ has to be compared with the experimental value of $0.4(1) \mathrm{eV}$. This shows that one has to improve the calculations of the very details of the level structure but the gross structure is already very well reproduced in our relativistic calculations.

\section{Conclusions}

The procedure, introduced in this work not only to calculate the energy of the molecule, but also to calculate the atomic total energies, has been found to increase the numerical accuracy. Otherwise it would have been almost impossible to evaluate potential energy curves which would be of direct relevance for chemical interpretation. Although the result for the general shape of the potential energy curve and the bond distance agree quite well with the experiment, a large amount of further emphasis has to be put into further refinement of this method and calculations with more extended basis sets.

\section{References}

1. Pyykkö, P.: Adv. Quantum Chem. 11, 353 (1978)

2. Fricke, B.: Structure and Bonding. Vol. 21, p. 89. Berlin, Heidelberg, New York: Springer 1975

3. Rosén, A., Ellis, D.E.: J. Chem. Phys. 62, 3039 (1975)

4. Ellis, D.E., Rosén, A.: Z. Physik A 283, 3 (1977)

5. Rosén, A.: Chem. Phys. Lett. 55, 311 (1978)

6. Rosén, A., Fricke, B.: Chem. Phys. Lett. 61, 75 (1979)

7. Rosén, A., Fricke, B., Morović, T., Ellis, D.E.: J. Phys. (Paris) 40, C4-218 (1979)

8. Koelling, D.D., Ellis, D.E., Bartlett, R.J.: J. Chem. Phys. 65, $3331(1976)$

9. Slater, J.C., Johnson, K.H.: Phys. Rev. B 5, 844 (1972)

10. Hartung, H., Fricke, B., Morović, T., Sepp, W.-D., Rosén, A. Phys. Lett. A 69, 87 (1978)

11. Bartlett, N.: Proc. Chem. Soc. 218 (1962)

12. Claassen, H.H., Selig, H., Malm, J.G.: J. Am. Chem. Soc. 84, 3593 (1962)

13. Bagus, P.S., Lin, B., Schaefer III, H.F.: J. Am. Chem. Soc. 94, 6635 (1972)

14. Bagus, P.S., Lin, B., Liskow, D.H., Schaefer III, H.F.: J. Am. Chem. Soc. 97, $7216(1975)$

15. Harshbarger, W., Bohn, R.K., Bauer, S.: J. Am. Chem. Soc. 89 , 6466 (1967)

16. Murchison, C., Reichman, S., Anderson, D., Overend, J., Schreiner, F.: J. Am. Chem. Soc. 90, 5690 (1968)

17. Reichman, S., Schreiner, F.: J. Chem. Phys. 5, 2355 (1969)

18. Bartlett, N., Sladky, F.O.: The Chemistry of Krypton, Xenon and Radon. University of California. Radiation Laboratory. Report 19658. June 1970

19. Pepkin, V.I., Lebedev, Y.A., Apin, A.Y.: Zh. Fiz. Khim. 43 1564 (1963)

20. Slater, J.C.: Phys. Rev. 34, 1293 (1929)

21. Slater, J.C.: Phys. Rev. 81, 385 (1951)

22. Ellis, D.E., Painter, G.S.: Phys. Rev. B 2, 2887 (1970)

23. Averill, F.W., Ellis, D.E.: J. Chem. Phys. 59, 6412 (1973)

24. Rosén, A., Ellis, D.E., Adachi, H., Averill, F.W.: J. Chem. Phys. 65, 3629 (1976)

25. Baerends, E.J., Ellis, D.E., Ros, P.: Chem. Phys. 2, 41 (1973)

26. Baerends, E.J., Ros, P.: Int. J. Quantum Chem. Symp. 12, 169 (1978)

27. Heijser, W., van Kessel, A.T., Baerends, E.J.: Chem. Phys. 16. 371 (1976)

28. Brundle, C.R., Jones, G.R.: Chem. Commun. 19, 1198 (1971)

29. Brundle, C.R., Robin, M.B., Jones, G.R.: J. Chem. Phys. 52, 3383 (1970)

R. Euler

B. Fricke

T. Morović

W.-D. Sepp

Fachbereich 18 - Physik

Gesamthochschule Kassel

Heinrich-Plett-Straße 40

D-3500 Kassel

Federal Republic of Germany

A. Rosén

Physics Department

Chalmers University of Technology

Göteborg

Sweden 